\title{
Evaluation of sonographic and strain elastographic findings in tubercular cervical \\ lymphadenopathy.Evaluation of sonographic and strain elastographic findings in tubercular cervical lymphadenopathy.
}

\section{RENU YADAV (D DOC.RENU0803@GMAIL.COM)}

Vardhman Mahavir Medical College and Safdarjung Hospital https://orcid.org/0000-0003-4559-0596

AMITA MALIK

Vardhman Mahavir Medical College and Safdarjung Hospital

RAJNI PRASAD

Vardhman Mahavir Medical College and Safdarjung Hospital

\section{Research Article}

Keywords: Sonography, Sonoelastography, Tubercular lymphadenitis, Strain ratio, Color coded elastogram

Posted Date: February 25th, 2022

DOI: https://doi.org/10.21203/rs.3.rs-1127425/v1

License: (9) This work is licensed under a Creative Commons Attribution 4.0 International License. Read Full License 


\section{Abstract}

Purpose-Tuberculous cervical lymphdaenopathy is the most common manifestation of extrapulmonary tuberculosis and frequently present imaging diagnostic dilemma with metastatic lymphadenopathy. Ours is observational study done to evaluate the role of sonography including Doppler and strain elastography in tubercular cervical lymphadenopathy.

Methods-100 fine needle aspiration cytology/histopathological examination(FNAC/HPE) proven tubercular lymph nodes were evaluated with sonography and strain elastography Features evaluated with sonography are location, size, short/long axis diameter(S/L) ratio, presence or absence of echogenic hilum ,intra-nodal necrosis, intra-nodal calcification associated soft tissue features like periadenitis or collection and pattern of vascularity. With strain elastography(USE) color coded elastograms and strain ratio were evaluated.

Results-On evaluation tubercular nodes are large size, $S / L$ ratio $>0.5$, show intra-nodal necrosis and peripheral vascular pattern. $91 \%$ tuberculous lymph nodes had color coded elastograms $>2$ pattern which is similar to malignant lymph nodes and $99 \%$ of tuberculous lymph nodes show strain ratio< 1.99 .

Conclusion - Grey scale and Doppler assessment of tubercular lymph nodes reveals findings similar to that encountered in metastatic nodes, hence differentiation difficult .While strain ratio being semiquantitative is more objective criteria of assessment compared to color coded elastograms. Hence adding semi-quantitative elastographic evaluation with US will help in further characterization of tuberculous nodes.

\section{Introduction}

Cervical lymphadenopathy is a common clinical entity with varied causes ranging from benign to malignant. Amongst benign causes, tuberculous lymphadenopathy is the most common etiology in endemic areas. ${ }^{1}$ Its incidence also increasing in developed countries with the increasing prevalence of acquired immunodeficiency syndrome (AIDS). Tuberculous lymphadenitis is the most common form of extrapulmonary tuberculosis. ${ }^{2}$ It is frequently bilateral with predilection for the posterior triangle of the neck. The involvement of lower cervical group of lymph nodes concomitantly increases the incidence of pulmonary involvement. ${ }^{3}$ There are three patterns of lymph node involvement. Imaging findings depends upon the stage of the disease at the time of examination. In the acute stage of tubercular granuloma, the lymph nodes are enlarged with homogeneous appearance. As the diseases progresses, the second pattern or the most common pattern of a suppurative node with central necrosis is seen. The third pattern is of a fibrocalcified node, frequently seen in chronic phase or treated patients. ${ }^{3,4,5}$ Proper diagnosis is required before initiating treatment due to prolonged and cumbersome drug regime. Radiological evaluation is performed in patients lacking the typical associated clinical features, nodes not amenable to clinical examination and patients not responding to treatment. 
FNAC/ biopsy remains the gold standard for final diagnosis of cervical lymphadenopathy. On histopathological examinatione majority of these nodes turn out to be benign, as malignancy accounts for less $1 \%$ of all cases of lymphadenopathy, ${ }^{6}$ hence an effective non-invasive imaging assessment can help preclude the need for invasive diagnostic procedures for obtaining a definite diagnosis.

Imaging modalities available for assessment of lymph nodes are Ultrasound (US), including color Doppler and sonoelastography, Computed Tomography(CT) and Magnetic Resonance Imaging(MRI).

Ultrasound is commonly used for evaluation of cervical lymphadenopathy as the nodes are superficial, easily accessible, non-invasive and free from risk of radiation. On US, a node is assessed for its location, size, shape, echogenecity, presence of intranodal necrosis, calcification and ancillary features, such as, matting and soft tissue edema. On Color Doppler, the pattern of vascularity was assessed.

CT and MRI helps to further characterize the sonographic abnormalities, confirm lymph nodes situated in deeper locations, with superior anatomical localization. However CT is associated with risk of radiation and MR studies are time consuming and require sedation in pediatric population. ${ }^{7}$

Ultrasound elastography (USE) has principle similar to the clinical method palpation and is a more objective method of assessment. The principle underlying strain elastography is that tissue compression produces a strain (displacement). This strain is seen to be lower in stiff tissues than in the softer tissues. ${ }^{8}$ Strain elastography is a very promising imaging modality for characterization of lymph nodes, especially the cervical lymph nodes which are easily accessible and effective compression can be applied using the transducer, against the underlying structures to obtain the color coded elastogram and strain ratio.

The current study aims to assess the utility of strain elastography in tuberculous nodes, which account for significant proportion of benign cervical lymphadenopathy.

\section{Material And Methods}

100 consecutive patients with tuberculous lymphadenitis confirmed on FNAC or histopathology with age $>5$ years were enrolled in the study after obtaining a written, informed consent. A lymph node with $>50 \%$ cystic area, presence of macro-calcifications or inadequate reference muscle tissue surrounding region of interest (ROI) were excluded from the study. In case of patient with multiple LNs, after ruling out exclusion criteria representative $L N$ with adequate surrounding reference tissue was taken up. History regarding onset, duration, progression, associated symptoms like fever, weight loss, change in voice was taken followed by general physical examination and local examination of enlarged lymph nodes. Local examination included assessment for distribution, size, consistency, fixity to overlying and underlying structures.

1. Sonography - Neck US was performed in both the transverse and longitudinal planes, with patients in supine position with the neck hyper-extended by placing a pillow under the patient's shoulders and lower neck for support. The scans were performed using Philips iU22 US scanner, equipped with 
linear array transducer of frequencies $5-17 \mathrm{MHz}$ and $3-9 \mathrm{MHz}$. Nodes were evaluated for their distribution, size, shape, nodal margins, presence or absence of echogenic hilum, echogenecity, presence of intranodal calcifications, intranodal reticulations and intranodal necrosis. Ancillary features such as matting and adjacent soft tissue edema were also assessed.

2. On color Doppler sonography vascular patterns were categorized as - Hilar, Avascular, Peripheral, Spotted, and Mixed.

3. Ultrasound elastography - was performed using Philips iU22 scanner equipped with linear array transducer of frequency $5-17 \mathrm{MHz}$ with strain compression elastographic capability. The elasticity image of the lymph node was evaluated qualitatively using color coded elastograms and semiquantitatively using strain ratio. Five patterns as described by Alam et al, were used for evaluation of color coded elastograms. The following patterns are described, with hard area displayed as red, soft as blue and tissues with intermediate strain as green:

Pattern 1 -absent or a very small hard area (red).

Pattern 2 - hard area (red) $<45 \%$ of the lymph node

Pattern 3 - hard area (red) $\geq 45 \%$

Pattern 4 -peripheral hard and central soft area

Pattern 5 - hard area (red) occupying entire lymph node with or without a soft rim.

The Strain ratio was calculated by the machine as ratio of strain of adjacent muscle to the strain of the lymph node.

Strain ratio>1.99 was calculated from receiver operating characteristics (ROC) curves plotted to differentiate benign from malignant lymph nodes (LNs) was used.

\section{Results}

\section{Demographic distribution -}

Age of the patients included in the study ranged from 7 -36yrs with mean age of 23.6yrs.

\section{Sonographic parameters -}

\section{B-mode sonography-}

LN Size $>8 \mathrm{~mm}$ was observed in all the patients. S/L ratio $>0.5$, intra-nodal calcification and loss of echogenic hilum was observed in $3 / 4^{\text {th }}$ of the population with intranodal necrosis was present in more than half of the studied LNs.(Table-1) 
Matting and soft tissue edema was seen in $30 \%$ of tubercular nodes.

\section{Color Doppler-}

$70 \%$ of the tubercular nodes showed peripheral vascular pattern.

\section{Sono-elastographic parameters -}

Color coded elastogram-

Color coded elastogram $\leq 3$ was observed in more than $91 \%$ of the LNs with approximately $3 / 4^{\text {th }}$ of the LNs show color coded elastogram pattern 3 while $9 \%$ showed pattern $>3$. (Table- 2$)$

Strain ratio $\leq 1.99$ was observed in $96 \%$ of the LNs while only $4 \%$ showed $>1.99$. ( Table-3)

\section{Discussion}

Tuberculous lymphadenitis is the most common form of extrapulmonary tuberculosis. ${ }^{2}$ It is versatile masquerader as it can resemble infection or neoplasm and poses a diagnostic challenge to the clinicians

${ }^{9}$ and on ultrasound and elastography. ${ }^{10}$

\section{B-mode sonography-}

Tubercular lymph nodes are large, $S / L$ ratio $>0.5$, shows absence of echogenic hilum, intranodal necrosis, calcification. Similar observations were made by Park J H et al. ${ }^{11}$ Ying et al ${ }^{12}$ in their study including 315 tuberculous nodes, reported that $79 \%$ had increased $S / L$ ratio $>0.5$ however the percentage was less than that in metastatic nodes $95 \%$. Absent hilus was reported in approx $76-86 \%$ of tuberculous nodes . Nodal calcification is late feature in tubercular lymphadenitis may help in diagnosis, however metastasis from papillary carcinoma thyroid may present with nodal calcification. Due to edema and periadenitis acoustic interface is blurred between nodes and surrounding tissues. Similar findings are also seen post radiotherapy. However in the absence of history these findings are highly suggestive of tubercular. ${ }^{13}$

\section{Color Doppler findings-}

Vascular pattern on color doppler imaging depends upon the degree of intranodal necrosis and stages of the disease. Cystic necrosis destroys the blood vessels of the lymph nodes resulting in avascular pattern, seen in $6-41 \%$ of tuberculous nodes . ${ }^{14,15}$, Avascularity may also be seen in later stages of the disease, when fibrosis and hyalinization cause compression or obliteration of intranodal vessels. ${ }^{16}$

Grey scale and Doppler assessment of tubercular lymph nodes reveals findings similar to that encountered in metastatic nodes, hence differentiation difficult. Further tuberculosis and metastasis are two major contributors to cervical lymphadenopathy .

\section{Strain elastography-}


Most of the tubercular nodes had color coded pattern of $\leq 3$ except $8 \%$ similar to metastatic LNs. Balasubrmaniam $S$ et al $^{17}$. in their study considered $\leq 2$ benign pattern while $>2$ malignant pattern. $83 \%$ tuberculous $L N s$ show $\leq 2$ pattern while $91 \%$ show $\leq 3$. They stated that tuberculous $L N$ s cannot be diagnosed on elastography due to overlapping features with both benign and malignant nodes. Tuberculous lymphadenitis with scarring calcification and necrosis may demonstrate stiffer areas. Similar findings were also observed by Ying L et al ${ }^{18}$. , Teng D K et al. ${ }^{19}$ and . Hasan D I et al. ${ }^{20}$

Strain ratio $\leq 1.99$ was observed in $99 \%$ of LNs except one. Hence strain ratio being semiquantitative assessment helps in differentiation of tuberculous lymph nodes from metastatic $L N$ s which demonstrate $S R>1.99$. Available literature on strain elastography in cervical lymphadenopathy shows strain ratio between 1.5 to 2.39 to differentiate benign from malignant lymphadenitis with no specific data on tubercular lymph nodes which are considered part of benign spectrum. ${ }^{21}$ Cut-off calculated from ROCs in our study to differentiate benign from malignant is within the available literature. $82.6 \%$ of tubercular lymph nodes show strain ratio $<1.5$, this increases to $95 \%$ with strain ratio $\leq 1.99$. Representative cases from our study are described in Fig 1,2 and 3.

Limitation-Strain elastography is free hand compression technique, with degree of compression can alter tissue stiffness, hence operator dependent. Ours was a retrospective study with inherent observer bias.

\section{Conclusion}

Strain ratio is a more objective criteria of assessment compared to color coded elastograms. Hence adding semi-quantitative strain elastographic evaluation with US may help in further characterization of lymph nodes above sonographic assessment.

\section{Abbreviations}

AIDS- Acquired immunodeficiency syndrome

CT- Computed Tomography

FNAC/HPE- fine needle aspiration cytology/histopathological examination

LNs- Lymph nodes

MRI- Magnetic Resonance Imaging

ROC -Receiver operating characteristics

ROI- Region of interest

SAD- Short axis diameter

S/L -short/long axis diameter 
US- Ultrasound

USE-Ultrasound elastography

\section{Declarations}

\section{Ethics approval and consent to participate -}

The study was approved by the ethical committee of Vardhman Mahavir Medical College \& Safdarjung Hospital, New Delhi. India 110029 with approval number IEC/VMMC/SJH/Thesis/November -2014/411 Written informed consent was obtained from all study subjects before enrolling into study.

Consent for publication- Not applicable.

Availablility of data and materials- Analyzed data is included in the article. Dataset used for the current study is available with the corresponding author and available on request.

Competing interests- None.

Conflict of interest- None

Funding - None

\section{Authors' contribution-}

Each author contributed substantially to the design of work. RY and AM collected, analyzed and interpreted the patient data generated from sonographic and sonoelastographic examination in cervical lymphadenopathy. RP performed the histopathological examination of FNAC/biopsy slides. RY and AM were major contributor in manuscript writing. All authors have read and approval the final manuscript and take full accountability of work and data given.

Acknowledgements - None

Consent to participate - Informed consent was obtained from all study participants.

\section{References}

1. Ochicha O, Edino ST, Mohammed AZ, Umar AB, Atanda AT. Pathology of peripheral lymph node biopsies in Kane, Northern Nigeria. Ann Afr Med 2007;6:104-108.

2. Marais BJ, Wright CA, Schaaf HS, Gie RP, Hesseling AC, Enarson DA et al. Tuberculous lymphadenitis as a cause of persistent cervical lymphadenopathy in children from a tuberculosis-endemic area. Pediatr Infect Dis J 2006;25:142-146.

3. Moon K W, Han M H, Chang K H, Im JG, Kim HJ, Sung K J et al. CT and MR imaging of Head and Neck Tuberculosis. Radiographics 1997;17:391-402. 
4. Som PM, Curtin HD, Mancuso AA. Imaging based classification for the cervical nodes designed as an adjunct to recent clinically based nodal classification. Arch Otolaryngol Head Neck Surg1999;125:388-396.

5. Eisenkraft BL, Som PM. The spectrum of benign and malignant etiologies of cervical node calcification. AJR1999;172:1433-1437.

6. Fijten $\mathrm{G} \mathrm{H}, \mathrm{Blijham} \mathrm{GH}$. Unexplained lymphadenopathy in family practice. An evaluation of the probability of malignant causes and the effectiveness of physicians workup. J Fam Pract.1988;27:373-376.

7. American College of Radiology. ACR Appropriateness Criteria: neck mass/adenopathy.American College of Radiology Website.www.acr.org/-/media/ACR/Documents/App Criteria/Diagnostic/Neck mass adenopathy.pdf.

8. Ying M, Bhatia KSS, Lee YP, Yuen HY, Ahuja AT. Review of ultrasonography of malignant neck nodes: grey scale, Doppler, contrast enhancement and elastography. Cancer imaging2013;13:658-669.

9. Levin-Epstein AA, Lucente FE. Scrofula the dangerous masquerader. Laryngoscope 1982;92:938-943

10. Abdelgawad E A, Abu-samra M F and Abdel -Azeem H M. B-mode ultrasound, color Doppler and sonoelastography in differentiation between benign and malignant cervical lymph nodes with special emphasis on sonoelastography. The Egyptian Journal of Radiology and Nuclear Medicine( 2020)51:157.

11. Park J H, Kim DW. Sonographic diagnosis of tuberculous lymphadenitis in the neck. J Ultrasound Med2014;33:1619-1626.

12. Ying M, Ahuja AT, Evans R, King W, Metreweli C. Cervical lymphadenopathy: sonographic differentiation between tuberculous nodes and nodal metastases from non-head and neck carcinomas. J Clin Ultrasound 1998;26:383-389.

13. Ahuja A, Ying M. Sonography of neck lymph nodes: Part II. Abnormal lymph nodes. Clin Radiol2003;58:359-366.

14. Na DG, Lim HK, Byun HS, Kim HD, Ko YH, Baek JH . Differential diagnosis of cervical lymphadenopathy: usefulness of color Doppler sonography. AJR Am J Roentgenol1997;168:13111316.

15. Wu CH, Chang YL, Hsu WC, Ko JY, Sheen TS, Hsieh FJ. Usefulness of Doppler spectral analysis and power Doppler sonography in the differentiation of cervical lymphadenopathies. AJR Am J Roentgenol1998;171:503-509.

16. Ahuja A, Ying M, Yuen YH, Metreweli C. Power Doppler sonography to differentiate tuberculous cervical lymphadenopathy from nasopharyngeal carcinoma. AJNR Am J Neuroradiol2001;22:735740 .

17. Balasubrmaniam S, Alagappan P, Ajis B A,Chelladurai A and Gnanasigamani S .Role of High Resolution Ultrasonogram and Elastography in Cervical Lymphadenopathy. IOSR Journal of Dental and Medical Sciences 2018;17:05-10. 
18. Ying L, Hou Y, Zheng HM, Lin X, Xie ZL, Hu YP. Real -time elastography for the differentiation of benign and malignant superficial lymph nodes: a meta-analysis. Eur J Radiol.2012;81:2576-2584.

19. Teng DK, Wang H, Lin YQ, Sui GQ, Guo F, Sun LN. Value of ultrasound elastography in assessment of enlarged cervical lymph nodes. Asian Pac J Cancer Prev.2012;13:2081-2085.

20. Hasan D I, Ahmed A F, Haggag R and Mohamed A M. Ultrasound elastography in pathological enlarged cervical lymph nodes compared to histopathology. The Egyptian Journal of Radiology and Nuclear Medicine 47(2016) 1349-59.

21. Young J C, Lee J H, Baek J H. Ultrasound elastography for evaluation of cervical lymph nodes. Ultrasonography 2015;34:157-164.

\section{Tables}

Table-1: Showing percentages of different sonographic parameters in studied tuberculous LNs.

\begin{tabular}{|ll|}
\hline Variables & Present (\%) \\
\hline Size $>\mathbf{8 m m}$ & 100 \\
\hline S/L ratio $>0.5$ & 74 \\
\hline Echogenic hilum & 74 \\
\hline Intranodal necrosis & 60 \\
\hline Intranodal calcification & 70 \\
\hline
\end{tabular}

Table-2: Showing color coded elastogram pattern in tuberculous LNs.

\begin{tabular}{|c|c|}
\hline Color coded elastogram & Tuberculous LNs(\%) \\
\hline $\mathbf{2}$ & 13 \\
\hline $\mathbf{3}$ & 78 \\
\hline $\mathbf{4}$ & 8.6 \\
\hline
\end{tabular}

Table -3: Showing strain elastogram pattern in tuberculous LNs. 


\begin{tabular}{|ll|}
\hline Strain ratio & Tuberculous $\mathrm{LNs}(\%)$ \\
\hline$<1$ & 57 \\
\hline $1-2$ & 39 \\
\hline $\mathbf{2}$ & 4 \\
\hline
\end{tabular}

\section{Figures}

\section{Figure 1}

Grey-scale1(A) and color Doppler 1(B) images of a 18 years old male patient shows enlarged node with $S A D 14 \mathrm{~mm}, S / L$ ratio 0.67 , heterogeneous echotexture with necrotic areas within and show displaced vascularity. Elastographic images 1 (C) of LN with color coded elastograms pattern 5 and strain ratio 1.02 .

\section{Figure 2}

Grey-scale2(A) and color Doppler2(B) images of 26 years old female patient shows $L N$ is well defined, has $S A D 1.2 \mathrm{~cm}, S / L$ ratio 0.62 and multiple punctate hyperchoic calcific foci. On color Doppler $L N$ shows peripheral vascularity. USE 2(C) image shows color coded elastogram shows pattern 3 and SR $=1.8$.

\section{Figure 3}

Grey-scale3(A) and Color Doppler 3(B) images of 8 year old boy shows the node is round with $S A D=$ $9.8 \mathrm{~mm}, \mathrm{~S} / \mathrm{L}$ ratio 0.8 and avascular pattern on color Doppler imaging. Elastographic images3(C) shows color coded elastograms pattern 4 and strain ratio 1.96.

\section{Supplementary Files}

This is a list of supplementary files associated with this preprint. Click to download.

- STROBEchecklistv4combinedPlosMedicineconverted.docx 